\title{
The p38 Mitogen-Activated Protein Kinase Is Involved in Associative Learning in Rabbits
}

\author{
Xuechu Zhen, Wei Du, Anthony G. Romano, Eitan Friedman, and John A. Harvey \\ Department of Pharmacology and Physiology, MCP Hahnemann University School of Medicine, Philadelphia, \\ Pennsylvania 19102
}

This study examined the role of the mitogen-activated protein kinase (MAPK) family during acquisition of the rabbit's classically conditioned eye-blink response. Eye-blink conditioning produced a significant, bilateral activation of both extracellular signal-regulated protein kinases (ERKs) and p38 MAPK in the anterior cerebellar vermis. There was also a significant bilateral activation of ERKs in the dorsal hippocampus with no change in p38 MAPK. These changes were seen at $2 \mathrm{~min}$ after the last conditioning session, were maintained for at least $180 \mathrm{~min}$, and occurred without any change in the protein expression of either ERKs or p38 MAPK. There were no changes in ERKs or p38 MAPK in frontal cortex, in cerebellar hemispheral lobule VI, or in a section of brainstem containing the inferior olive. Moreover, the stress-related protein kinase Jun N-terminal kinase (JNK), another subfamily of MAPKs, was not altered in any of the brain regions examined. Animals receiving explicitly unpaired presen-

Understanding the mechanisms of neuronal plasticity that are associated with learning and memory remains a challenge in neurobiology. It has been assumed that memory consolidation requires regulation at the level of gene and protein expression (Impey et al., 1999). Recent studies have indicated that mitogenactivated protein kinases (MAPKs) may play a central role in the development of synaptic plasticity underlying learning and memory (Kornhauser and Greenberg, 1997; Impey et al., 1999; Orban et al., 1999). There are three subfamilies of MAPKs including the extracellular signal-regulated protein kinases (ERKs), Jun N-terminal kinase (JNK), and p38 MAPK. These MAPKs are activated by different upstream cascades and are involved in the regulation of distinct nuclear transcriptional factors (Davis, 1993). ERKs and p38 MAPK are expressed abundantly in the CNS, especially in areas that are thought to be involved in learning and memory such as the hippocampus and cerebellum (Ortiz et al., 1995; Zhen et al., 1999; Lee et al., 2000). In the rat, activation of ERKs in the hippocampus was reported to be essential for long-term spatial memory as measured in the water maze (Blum et al., 1999) and in memory consolidation for fear conditioning (Atkins et al., 1998; Cammarota et al., 2000). Acti-

Received Sept. 25, 2000; revised May 3, 2001; accepted May 8, 2001.

This work was supported by United States Public Health Service Grants MH16841 and DA11164 to J.A.H. and Grant DA11029 to E.F.

Parts of this paper have been presented previously at the annual meeting of the Society for Neuroscience (Nov. 4-9, 2000; New Orleans, LA).

Correspondence should be addressed to Dr. Xuechu Zhen, Department of Pharmacology and Physiology, MCP Hahnemann University School of Medicine, 245 North 15th Street, NCB, Mail Stop \# 488, Philadelphia, PA 19102. E-mail: xuechu.zhen22@drexel.edu.

Copyright (C) 2001 Society for Neuroscience $0270-6474 / 01 / 215513-07 \$ 15.00 / 0$ tations of a conditioned stimulus and an unconditioned stimulus did not acquire conditioned responses (CRs) and did not demonstrate any changes in ERKs, p38 MAPK, or JNK. The intraventricular injection of SB203580, a selective p38 MAPK inhibitor, significantly retarded CR acquisition and blocked the learning-related increases in p38 MAPK activity in the anterior vermis. PD98059, a selective MAPK kinase inhibitor, had a smaller and only marginally significant effect on CR acquisition, although it did block the learning-related increases in ERK activity in both the hippocampus and anterior vermis. These results indicate that $\mathrm{p} 38 \mathrm{MAPK}$ is activated during associative learning and may play a role in the transcriptional events that lead to memory consolidation.

Key words: signal transduction; MAPKs; associative learning; synaptic plasticity; cerebellum; hippocampus

vation of MAPK in the cerebral cortex was reported to be associated with taste-aversion learning in the mouse (Swank, 2000b). The pharmacological antagonism of MAPKs was also shown to impair spatial learning in the rat (Blum et al., 1999; Selcher et al., 1999), fear conditioning in the rat (Atkins et al., 1998; Schafe et al., 1999), and taste-aversion learning in the mouse (Swank, 2000a). Evidence has also been presented suggesting that long-term memory may involve a specific and differential activation of the different families of MAPKs. For example, rats that had developed a long-term memory of a specific taste demonstrated a differential activation of ERKs and JNK but not p38 MAPK in the insular cortex when exposed to a novel taste (Berman et al., 1998). The studies cited above suggest that, depending on the learning task used, various brain areas and various subfamilies of MAPKs may become involved in long-term learning and memory.

Classical conditioning of the rabbit's eye-blink response as measured by either extension of the nictitating membrane or external eyelid closure has been widely accepted as a reliable procedure for the study of associative learning in both human and nonhuman subjects (Gormezano et al., 1983; Harvey, 1987). The signaling mechanisms underlying the neuronal plasticity involved in this form of associative learning remain unclear. However, microinfusion of a protein kinase inhibitor into the cerebellum attenuated acquisition of conditioned eye-blinks in the rabbit (Chen and Steinmetz, 2000), suggesting a role of the protein kinases in mediating eye-blink conditioning. The present study was designed to examine in greater detail the role of MAPKs in associative learning in the rabbit. We found that ERKs and p38 MAPK are differentially activated in dorsal hippocampus and in 
cerebellar vermis during acquisition. Furthermore, our data present the first evidence that p38 MAPK may play an important role in associative learning.

\section{MATERIALS AND METHODS}

Subjects. New Zealand White male rabbits weighing 1.8-2.2 kg (Covance, Inc., Denver, PA) were housed individually on a $12 / 12 \mathrm{hr}$ light/dark cycle in an American Association for Accreditation of Laboratory Animal Care-approved colony at $22 \pm 1{ }^{\circ} \mathrm{C}$ with rabbit chow and water available ad libitum. Rabbits were given $5 \mathrm{~d}$ of adaptation to the facility before the start of experiments. All animal experiments were performed in accordance with the National Institutes of Health (NIH) guide Principles of Laboratory Animal Care (NIH publication number 85-23; revised 1985).

Materials. PD98059 was obtained from Calbiochem (La Jolla, CA), and SB203580 was provided by SmithKlein (King of Prussia, PA). Myelin basic protein (MBP) and c-Jun (169) glutathione $S$-transferase (GST) were obtained from Upstate Biotechnology (Lake Placid, NY). Electrophoresis reagents were obtained from Bio-Rad (Richmond, CA). ERK2, anti-JNK1, and anti-p38 MAPK were purchased from Santa Cruz Biotechnology (Santa Cruz, CA). Horseradish peroxidase-linked secondary antibodies were obtained from Pierce (Rockford, IL). [ $\left.\gamma-{ }^{32} \mathrm{P}\right]$ ATP (3000 $\mathrm{Ci} / \mathrm{mmol}$ ) was purchased from NEN (Boston, MA). Other reagents were purchased from standard laboratory suppliers.

Behavioral apparatus. The conditioning apparatus including the computer hardware and ASYST software for stimulus control and data acquisition has been described in detail elsewhere (Romano et al., 1991). Movements of the right nictitating membrane (NM) were measured by a potentiometer and digitized every $5 \mathrm{msec}$ with a resolution of $0.03 \mathrm{~mm}$ of NM movement per analog-to-digital count. A response was defined as a $0.50 \mathrm{~mm}$ or greater extension of the $\mathrm{NM}$, and its onset latency was calculated from the time at which the response first deviated from baseline by at least $0.03 \mathrm{~mm}$. The $100 \mathrm{msec}$ air puff unconditioned stimulus (US) was set at a pressure of $200 \mathrm{gm} / \mathrm{cm}^{2}$ as measured at the end of a metal tube placed 5-7 $\mathrm{mm}$ from the center of the right cornea. The conditioned stimulus (CS) was a $200 \mathrm{msec}, 1 \mathrm{kHz}, 90 \mathrm{~dB}$ tone CS delivered through a speaker located in front of and above the rabbit. Before each experiment, animals were placed in the sound-attenuated conditioning chambers containing a house light for a 60 min adaptation session, during which no stimuli were presented and no drugs were administered. Conditioning sessions began on the day after this adaptation session.

Conditioning procedures. Each conditioning session consisted of 60 paired presentations of the CS and US at an average intertrial interval of $60 \mathrm{sec}$ (range, $55-65 \mathrm{sec}$ ). There was one such conditioning session each day for $3 \mathrm{~d}$. In each conditioning trial, onset of a tone CS was followed $200 \mathrm{msec}$ later by offset of the tone CS and the simultaneous onset of the $100 \mathrm{msec}$ air puff US. Responses were scored as conditioned responses (CRs) if they occurred within the $200 \mathrm{msec}$ after CS onset and as unconditioned responses (URs) if they occurred after US onset. To assess the occurrence of nonassociative learning, a separate group of animals was exposed to three daily sessions consisting of the explicitly unpaired presentations of the CS and US. In this procedure, animals received a pseudorandom presentation of $60 \mathrm{CS}$-alone and $60 \mathrm{US}$-alone trials at an intertrial interval of $30 \mathrm{sec}$ (range, 25-35 sec). All other parameters were the same as in the paired CS-US acquisition procedure. The paired CS-US and the unpaired stimulus procedures were performed concurrently.

Learning-induced changes in MAP kinases. In a first study, three groups of rabbits were used to measure the learning-induced, sustained activation of MAPKs in four brain regions. One group received $3 \mathrm{~d}$ of paired CS-US presentations, and the second group received $3 \mathrm{~d}$ of unpaired CS and US presentations. The third group consisted of controls that remained undisturbed in their home cages during these $3 \mathrm{~d}$ to provide a measure of basal levels of the MAPKs. At the end of the third day of conditioning, all animals were returned to their home cages; $180 \mathrm{~min}$ later they were decapitated, and the brains were dissected to obtain samples of left and right frontal cortex, dorsal hippocampus, and anterior cerebellar vermis as well as a bilateral section of brainstem containing the inferior olive. Brain samples were frozen on dry ice and stored at $-70^{\circ} \mathrm{C}$ until the time of assay. A second study used two groups of rabbits to examine the time course for the learning-induced activation of MAPKs. One group received $3 \mathrm{~d}$ of paired CS-US conditioning, whereas the second group consisted of controls that had remained in their home cages during these $3 \mathrm{~d}$. Samples of left and right anterior cerebellar vermis, hemispheral lobule VI, and dorsal hippocampus were obtained at 2 and 30 min after the end of the third conditioning session and from the experimentally naïve controls. Samples were frozen on dry ice and stored at $-70^{\circ} \mathrm{C}$ until assay.

Effect of MAPK inhibitors on learning and on MAPK activation. All animals were anesthetized with sodium pentobarbital, and a guide cannula-obturator assembly (Welsh and Harvey, 1991) was implanted into the left lateral ventricle as described previously (Aloyo et al., 1993). Rabbits were allowed $5 \mathrm{~d}$ of postsurgical recovery before beginning behavioral training. PD98059 and SB203580 were suspended in DMSO at a concentration of $40 \mu \mathrm{g} / 7.5 \mu \mathrm{l}$. Rabbits were injected with DMSO vehicle $(n=19)$, PD98059 $(n=17)$, or SB203580 $(n=11)$ into the left lateral ventricle in a volume of $7.5 \mu \mathrm{l}$ delivered in $1 \mathrm{~min}$. The cannula then remained in place for an additional minute before being removed and replaced with the obturator. Drug or vehicle injections occurred 15-20 min before each of the three conditioning sessions. At the end of the third conditioning session, four vehicle controls, six SB203580injected animals, and six animals injected with PD98059 as well as six animals that had been allowed to remain undisturbed in their home cages to provide a measure of basal levels of the MAPKs were decapitated, and the dorsal hippocampus and anterior vermis were then collected for assay of the activity of MAPK kinases. Another group of animals that had been injected with SB203580 $(n=5)$ and their vehicle controls $(n=5)$ were allowed to sit undisturbed in their home cages for $3 \mathrm{~d}$. On the third day they were given a 60 min testing session under extinction conditions during which there were $60 \mathrm{CS}$-alone presentations.

Preparation of protein extracts. Stored brain tissues were homogenized in $10 \mathrm{vol}$ of lysis buffer (buffer A) containing $50 \mathrm{~mm}$ Tris HCl, pH 7.4, 150 $\mathrm{mm} \mathrm{NaCl}, 20 \mathrm{~mm} \beta$-glycerophosphate, $1 \mathrm{~mm}$ EGTA, $20 \mathrm{~mm} \mathrm{NaF}, 3 \mathrm{~mm}$ $\mathrm{Na}_{3} \mathrm{VO}_{4}, 1 \mathrm{~mm}$ PMSF, $2 \mu \mathrm{g} / \mathrm{ml}$ aprotinin and leupeptin, and $1 \% \mathrm{NP}-40$. Lysates were centrifuged at $12,000 \times \mathrm{g}$ for $15 \mathrm{~min}$ at $4^{\circ} \mathrm{C}$ to precipitate the debris, and the protein content in the supernatant was determined by the protein assay kit of Bio-Rad. Aliquots of supernatants were used in the immunoprecipitation or immunoblotting experiments described below.

In vitro immune complex kinase assays. For immunoprecipitation, lysate protein $(500 \mu \mathrm{g}$ for ERKs, $300 \mu \mathrm{g}$ for JNK, and $100 \mu \mathrm{g}$ for p38 MAPK) was incubated with respective antibody overnight at $4^{\circ} \mathrm{C}$ before the addition of $15 \mu \mathrm{l}$ of protein A/G-PLUS agarose (Santa Cruz Biotechnology) and incubation for an additional $2 \mathrm{hr}$ at $4^{\circ} \mathrm{C}$ (the ERK2 antibody reacted with ERK2 but also reacted slightly with ERK1). The immune complex was washed three times with buffer A and twice with the corresponding kinase assay buffer. ERK and p38 MAPK activity analysis was conducted for $20 \mathrm{~min}$ at $30^{\circ} \mathrm{C}$ in the presence of $50 \mu \mathrm{M}$ $\left[\gamma^{-32} \mathrm{P}\right] \mathrm{ATP}(5 \mu \mathrm{Ci})$ and $0.2 \mathrm{mg} / \mathrm{ml} \mathrm{MBP}$ as described previously (Zhen et al., 1998, 1999). The reaction was terminated by spotting $10 \mu \mathrm{l}$ of reaction mixture onto $\mathrm{p} 81$ paper. Filters were washed five times for $5 \mathrm{~min}$ each in $0.85 \% \mathrm{H}_{3} \mathrm{PO}_{4}$ followed by rinsing in acetone for $5 \mathrm{~min}$. Radioactivity incorporated into substrates was determined by scintillation counting. In some experiments, aliquots of reaction mixture were boiled with an equivalent vol of $2 \times$ sample buffer, and the products were resolved by SDS-PAGE. The gels were stained with Coomassie blue to confirm the equivalence of loaded substrate. Phosphorylated c-Jun/BMP was assessed by autoradiography.

Immunoblotting. Lysate protein ( $20 \mu \mathrm{g} / \mathrm{lane})$ was separated by SDS gel electrophoresis and transferred to nitrocellulose membranes. The blots were probed with their respective antibody. The signals were visualized with the Supersignal Western Blot Detection System (Pierce).

Data analysis. Behavioral data were subjected to a repeated-measures analyses of variance using SYSTAT 7.0 for Windows (SPSS, Inc., Chicago, IL). Follow-up tests of significant effects were performed using Dunnett's $t$ test. Protein kinase data were expressed as the mean \pm SEM and were analyzed by ANOVA followed by Newman-Keuls test. The significance for all comparisons was set at $p<0.05$.

\section{RESULTS}

\section{ERKs and p38 MAPK are differentially activated in the dorsal hippocampus and anterior cerebellar vermis of animals demonstrating associative learning}

Rabbits receiving paired presentations of CS and US demonstrated a rapid and significant $(p<0.001)$ acquisition of CRs to the tone CS (Fig. 1). This acquisition of CRs was caused by associative learning because the unpaired presentation of CS and 


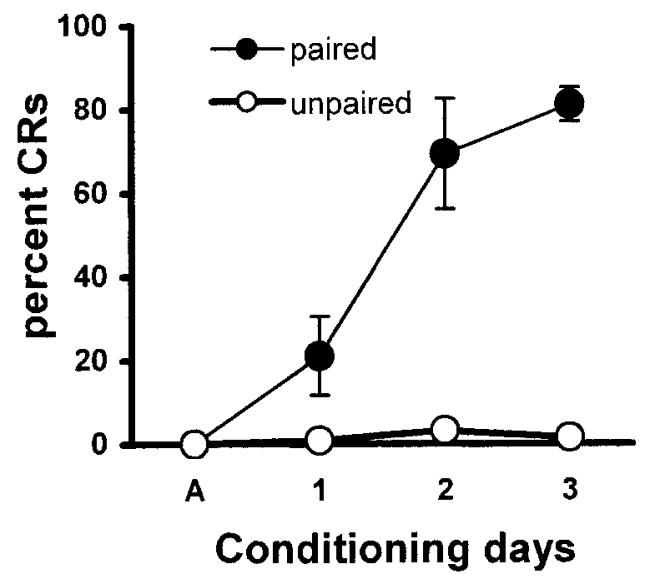

Figure 1. Acquisition of CRs to the tone CS during delay eye-blink conditioning. Data are expressed as the mean percentage of CS-elicited responses for rabbits receiving $3 \mathrm{~d}$ of either paired CS-US training (paired; $n=4$ ) or explicitly unpaired presentations of CS and US (unpaired; $n=4$ ). The points above $A$ are the percentage of responses during an adaptation session when no stimuli were presented. Error bars represent \pm 1 SEM.

US had no significant effect on responding during the CS. On the last day of conditioning, the mean percentage of CS-elicited responses was significantly $(p<0.001)$ greater in the paired CS-US group $(81.1 \pm 4.1 \%)$ compared with the unpaired controls $(1.4 \pm 0.9 \%)$. As shown in Figure 2, animals that had received paired CS-US conditioning and had demonstrated significant associative learning also demonstrated a significant and selective elevation of ERKs and p38 MAPK at 180 min after the third conditioning session. Both ERKs and p38 MAPK were significantly elevated in cerebellar vermis at this time (Fig. $2 A$ ), whereas only ERKs were significantly elevated in the dorsal hippocampus (Fig. 2B). These changes in dorsal hippocampus and cerebellar vermis were caused by associative learning because animals that had received unpaired CS and US presentations and had failed to demonstrate any CS-elicited responses also failed to demonstrate any changes in ERKs or p38 MAPK in either the anterior cerebellar vermis (Fig. $2 A$ ) or dorsal hippocampus (Fig. $2 B)$. There were no learning-induced changes in ERKs or p38 MAPK in the frontal cortex (Fig. 2C) or the inferior olive (Fig. $2 D$ ). JNK, a stress-activated MAP kinase, was not altered in any brain region of the paired CS-US or unpaired CS and US groups compared with normal controls (Fig. $3 A$ ). The learning-induced increases in ERKs and p38 MAPK observed in the anterior cerebellar vermis and dorsal hippocampus occurred without any change in either ERK or p38 MAPK protein expression in the three experimental groups (Fig. 3B).

Time course for learning-induced changes in ERK and p38 MAPK activity in the dorsal hippocampus and anterior cerebellar vermis

Rabbits receiving paired presentations of CS and US $(n=7)$ had achieved a mean of $84.4 \pm 8.7 \%$ CRs by the third day of conditioning. A significant learning-induced increase in ERK activity of both the right and left dorsal hippocampus was observed at 2 min after the third conditioning session, and this increase was maintained at 30 and $180 \mathrm{~min}$ (Fig. 4A,B). There were no differences between the activation observed in the left and right dorsal hippocampus at any time point. In contrast, p38 MAPK activity was not increased in either the left or right dorsal hippocampus at

\section{A: cerebellar vermis $\quad B$ : dorsal hippocampus}

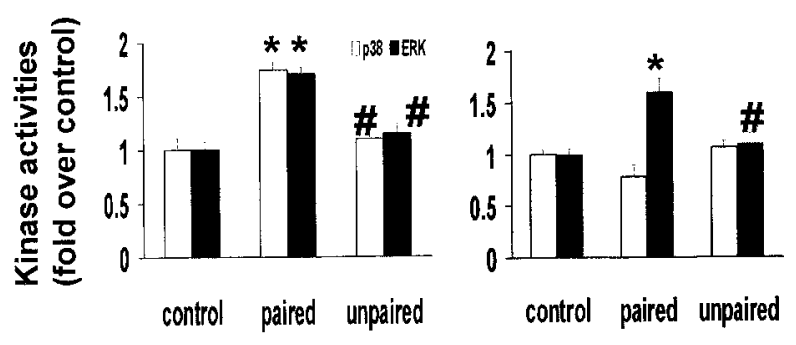

\section{C: frontal cortex}

D: inferior olive

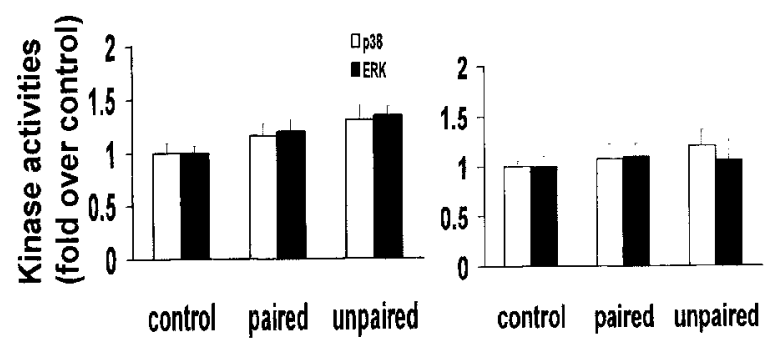

Figure 2. Learning-related activation of MAPKs in four brain regions. Animals of Figure 1 were decapitated at 180 min after the third conditioning day, and activities of MAPKs were measured by in vitro immune complex kinase assay using MBP as substrate. The radioactivity incorporated into MBP was determined by scintillation counting. The results are presented as a ratio of the values obtained in normal controls that had not been exposed to any conditioning procedure (see Materials and Methods). Each mean is based on four to seven determinations and represents values for the left hippocampus $(B)$, frontal cortex $(C)$, left cerebellar vermis $(A)$, and a bilateral section of brainstem containing the inferior olive $(D)$. Error bars represent \pm 1 SEM $\left({ }^{*} p<0.05\right.$ compared with control; \#p $<0.05$ compared with paired CS-US group).

any of these time points. The increase in ERK and p38 MAPK activity of both the right and left cerebellar vermis was also significant at 2 min after the third conditioning session, and this increase was also maintained at both 30 and $180 \mathrm{~min}$ (Fig. 4C,D). Again, there were no differences between the left and right anterior vermis. We also examined ERK and p38 MAPK activity in hemispheral lobule VI at 2 and $30 \mathrm{~min}$ after the last conditioning session. In contrast with the results obtained in the cerebellar vermis, there were no changes in either ERKs or p38 MAPK in hemispheral lobule VI of the cerebellum at either the 2 or 30 min time interval (Fig. 5).

Intraventricular p38 MAPK inhibitor impairs acquisition but not performance of eye-blink conditioning in rabbits

Administration of the p38 MAPK inhibitor SB203580 induced a dramatic attenuation of CR acquisition (Fig. 6), whereas the selective MAPK kinase (MEK) inhibitor PD98059 produced a small but consistent retardation. Vehicle-injected animals reached $82.6 \pm 4.4 \%$ CRs by the last day of conditioning, compared with $71.6 \pm 7.0 \%$ for animals injected with PD98059 and $48.0 \pm 10.4 \%$ for animals injected with SB203580. There was a significant effect of drug treatment $\left(F_{(2,44)}=4.96 ; p<0.05\right)$, and a follow-up Dunnett's test indicated that SB203580 had produced 


\section{A: JNK activity}
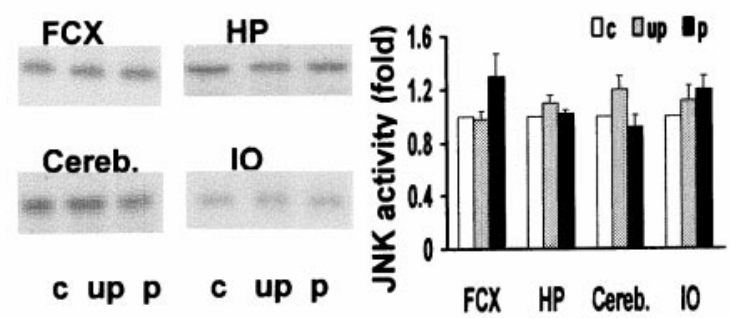

B: protein levels of MAPKs

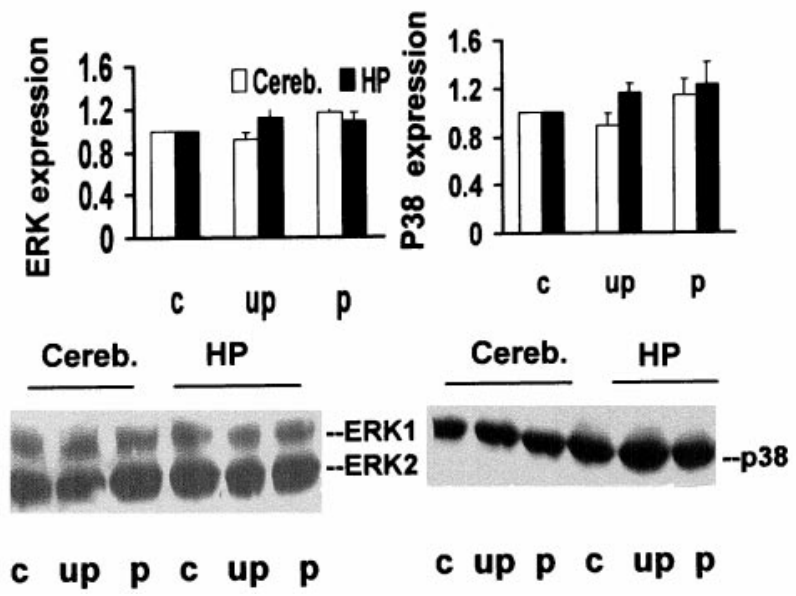

Figure 3. Conditioning does not alter JNK activity and MAPK protein levels. $A$, Representative autoradiographs of JNK activities in various brain areas (left) and summary data of densitometric scans (in relative density units) obtained from at least four animals in each group (right). $B$, Summary of densitometric scans for ERK2 (top left) and p38 MAPK (top right) expression and representative Western blots of ERK (bottom left) and p38 MAPK (bottom right) protein levels. Tissue was obtained from controls and from animals of Figure 1 that had been decapitated at 180 min after the last training session. JNK activity was measured by in vitro immune complex kinase assay using c-Jun GST as substrate. The radioactivity incorporated into c-Jun was determined by autoradiography. ERK and p38 MAPK protein levels were detected by Western blotting using anti-ERK2 and p38 MAPK antibody, respectively. The experiments of $A$ and $B$ were repeated three to four times with similar results. $c$, Control; Cereb., anterior vermis of cerebellum; $F C X$, frontal cortex; $H P$, dorsal hippocampus; $I O$, section of brainstem containing the inferior olive; $p$, paired; up, unpaired.

a significant retardation of learning $(p<0.01)$. There was also a significant days effect $\left(F_{(2,88)}=127.81 ; p<0.001\right)$ and a marginally significant drug-by-days interaction $\left(F_{(4,88)}=2.38 ; p=\right.$ $0.058)$, suggesting that the SB203580-treated animals were acquiring CRs at a slower rate than were controls. In contrast, the retardation of CR acquisition produced by PD98059 was not significant $(p<0.15)$, although a day-by-day comparison with vehicle controls indicated a marginally significant decrease in CRs on day $2(p=0.074)$. SB203580 not only produced a retardation of learning but also blocked the learning-related increases in p38 MAPK activity in the left anterior vermis (Fig. 7, top). PD98059 completely blocked the learning-related activation of ERK activity in both the hippocampus and vermis (Fig. 7, bottom), although
A: right dorsal hippocampus

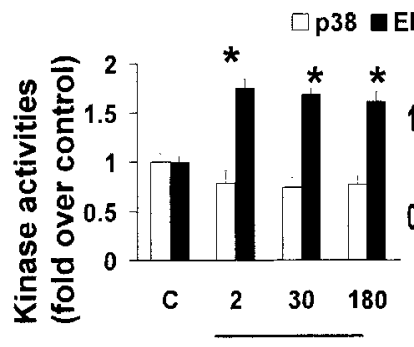

Time (min)

\section{B: left dorsal hippocampus}

\section{RK}

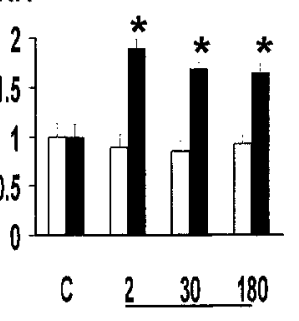

Time (min)

\section{C: right anterior cerebellar vermis}

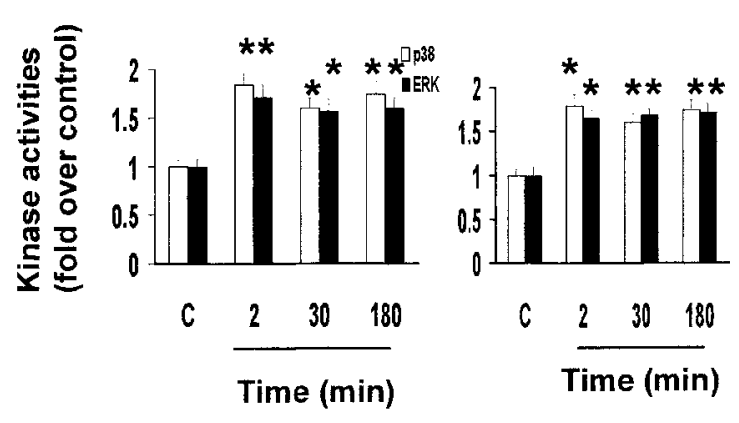

Figure 4. Time course for learning-induced ERK and p38 MAPK activation. Animals were decapitated at designated times after the last conditioning session. ERK and p38 MAPK activities were determined in both the left $(B)$ and right $(A)$ dorsal hippocampus and anterior vermis of the cerebellum $(C, D)$ as described in Figure 2. The results are presented as a ratio of the values obtained in normal controls $(C)$ that had not been exposed to any conditioning procedure. Each value is the mean $\pm 1 \mathrm{SEM}$ of determinations obtained from three to nine animals $\left({ }^{*} p<0.05\right.$; ${ }^{* *} p<$ 0.01, compared with control).

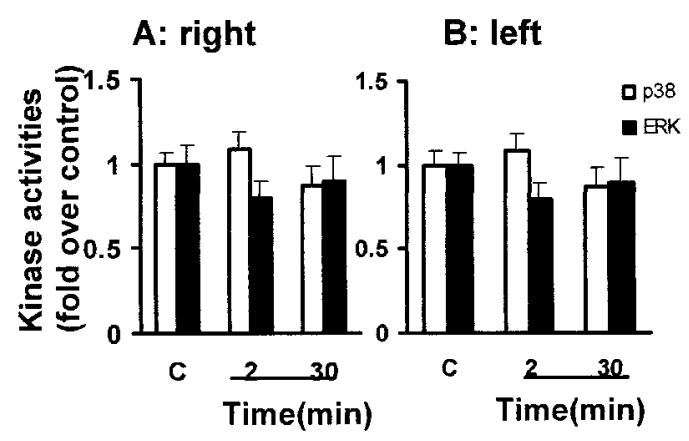

Figure 5. Associative learning does not induce MAPK activation in hemispheral lobule VI of the cerebellum. The ERK and p38 MAPK activities in hemispheral lobule VI (right, $A$; left, $B$ ) were measured at 2 and $30 \mathrm{~min}$ after the last conditioning session. Data are presented as described in Figures 2 and 4, and each value is the mean of three to nine determinations.

it had produced a much smaller retardation of $\mathrm{CR}$ acquisition (see Fig. 6). Similar inhibitory effects were also observed in the right hippocampus and vermis (data not shown).

The retardant effects of SB203580 on CR acquisition were 


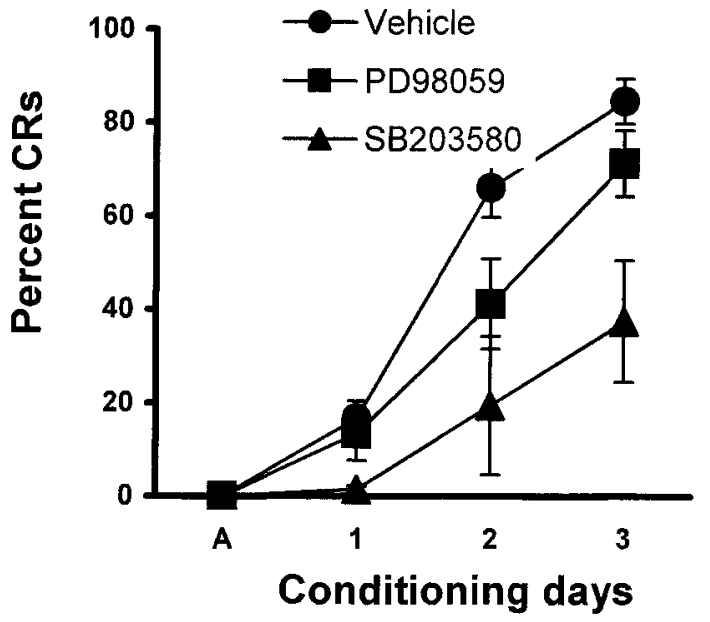

Figure 6. The p38 MAPK inhibitor SB203580 attenuates acquisition of CRs. Rabbits were injected with DMSO vehicle $(n=19), 40 \mu \mathrm{g}$ of SB203580 $(n=11)$, or $40 \mu \mathrm{g}$ of PD98059 $(n=17)$ into the left lateral ventricle 15-20 min before each of the three conditioning sessions. Data are presented as the mean percentage of CRs. The points above $A$ are the percentage of responses during an adaptation session when no stimuli were presented. Error bars represent \pm 1 SEM.
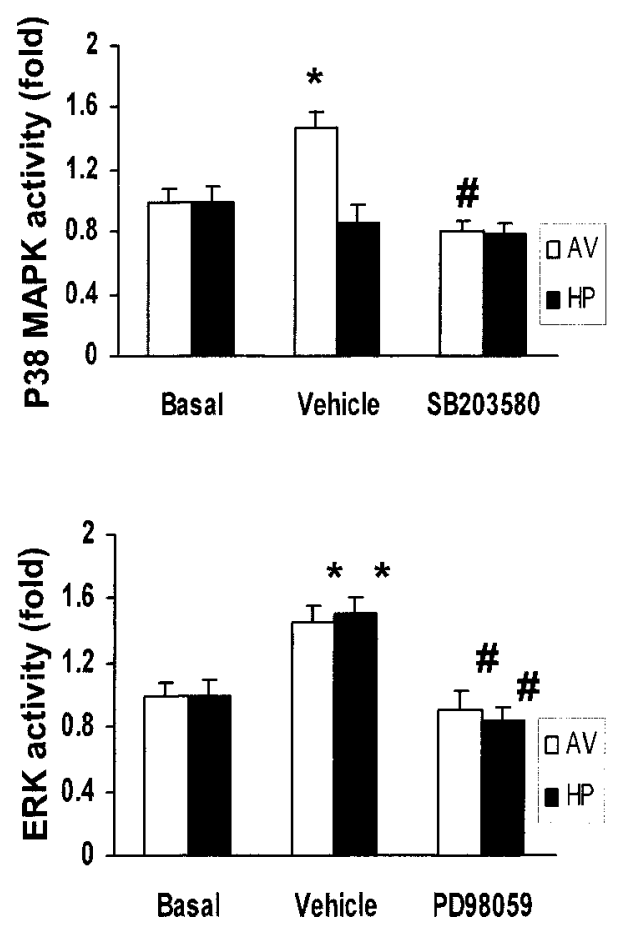

Figure 7. Intraventricular administration of MAPK inhibitors blocked the learning-associated activation of MAPKs. Rabbits were injected with DMSO or inhibitors as described in Figure 6. Animals were decapitated at 2 min after the last conditioning session. ERK (bottom) and p38 MAPK (top) activities were determined in both the left $(H P)$ and right (data not shown) dorsal hippocampus and anterior vermis of the cerebellum $(A V)$ as described in Figure 2. The results are presented as a ratio of the values obtained in basal controls that had not been exposed to any conditioning procedure. Each value is the mean \pm 1 SEM of determinations obtained from four to eight animals $\left({ }^{*} p<0.01\right.$ compared with basal; $\# p<0.01$ compared with vehicle injection).

further examined for possible effects of the drug on performance variables (Table 1$)$. At the end of the third conditioning session, the animals that had been injected with SB203580 $(n=5)$ and

\begin{tabular}{|c|c|c|c|}
\hline & $\begin{array}{l}\text { Vehicle } \\
(n=5)\end{array}$ & $\begin{array}{l}\text { SB203580 } \\
(n=5)\end{array}$ & $p$ value \\
\hline $\begin{array}{l}\% \text { CRs (Last day of } \\
\text { conditioning) }\end{array}$ & $83.9 \pm 9.4$ & $37.6 \pm 13.0$ & $p<0.05$ \\
\hline \% CRs (Extinction day) & $75.1 \pm 9.8$ & $30.3 \pm 8.2$ & $p<0.05$ \\
\hline
\end{tabular}

their vehicle controls $(n=5)$ were allowed to sit undisturbed in their home cages for $3 \mathrm{~d}$. On the third day, they were given an extinction session but were not injected with drug or vehicle. The percentage of CRs demonstrated by vehicle control animals during the extinction session $(75.1 \%$ ) was not significantly different from the percentage of CRs that they had demonstrated on the last day of conditioning $(83.9 \%)$. Similarly, the animals that had received SB203580 and had achieved only $37.6 \%$ CRs on the last day of conditioning demonstrated an equivalent percentage of CRs $(30.3 \%)$ during the extinction session. Finally, the percentage of CRs demonstrated by the SB203580 group was significantly lower than that of vehicle controls on both the last day of conditioning and the day of extinction (Table 1). To examine further the potential effects of SB203580 on performance variables, we compared the amplitude of URs during an early 10 trial block of CS-US pairings when no learning was detected. The UR amplitude of the 11 animals receiving SB203580 averaged $3.4 \pm 0.6$ $\mathrm{mm}$, which was not significantly different from the average of their 8 vehicle controls $(4.5 \pm 1.0 \mathrm{~mm})$.

In summary, ERK activity was elevated in both the dorsal hippocampus and vermal cerebellum as a consequence of associative learning, whereas p38 MAPK activity showed an associative learning-dependent increase only in the anterior vermis of the cerebellum. Furthermore, an inhibitor of p38 MAPK activity significantly retarded the rate of associative learning and prevented the learning-related increases in p38 MAPK activation. An MEK inhibitor prevented the increase in ERKs in the hippocampus and vermis but produced only a small and marginally significant retardation of learning.

\section{DISCUSSION}

The learning-dependent activation of ERKs and P38 MAPK is selective and sustained

Our results indicate that there is an associative learningdependent activation of MAPKs in the dorsal hippocampus and cerebellar vermis in rabbits. A bilateral activation of ERKs was observed in the dorsal hippocampus and in the anterior cerebellar vermis at 2 min after a third conditioning session and was sustained for up to $180 \mathrm{~min}$ after conditioning. In addition, we observed a novel bilateral activation in p38 MAPK in the anterior cerebellar vermis that had the same time course as that of the ERKs. These changes in ERK and p38 MAPK activities were not caused by any change in total ERK or p38 MAPK protein. The activation of MAPKs was only seen in animals demonstrating associative learning. Animals receiving explicitly unpaired presentations of stimuli failed to demonstrate any acquisition of CS-elicited responses and also failed to demonstrate any activation of ERKs in the dorsal hippocampus or ERKs and p38 MAPK in the vermal cerebellum. Thus, the changes in MAPK activities were not simply caused by stimulus presentation, sensitization, or pseudoconditioning but were dependent on the acquisition of associative learning. Moreover, the activation in 
ERKs and p38 MAPK occurred without any changes in JNK, a well known stress-related MAP kinase. Finally, these changes in MAPK activities were region specific because the prefrontal cortex, cerebellar hemispheral lobule VI, and a section of brainstem containing the inferior olivary nucleus failed to demonstrate any changes in activities of ERKs, p38 MAPK, or JNK.

\section{Significance of activation of ERKs in the dorsal hippocampus}

The hippocampus is importantly involved in spatial learning (Morris et al., 1982), in cued but not contextual fear conditioning (Phillips and LeDoux, 1994), and in trace but not delay eye-blink conditioning (Moyer et al., 1990; Weiss et al., 1999). Our findings of a selective activation of ERKs in the dorsal hippocampus are in agreement with the findings of several investigators who reported increases in ERK activity in the dorsal hippocampus of rats that had been trained in a spatial memory task using the Morris water maze (Blum et al., 1999) and in memory consolidation for fear conditioning (Atkins et al., 1998; Cammarota et al., 2000). However, the relevance of ERK activation in the hippocampus to associative learning is far from clear. For example, the increase in ERK activation in the dorsal hippocampus in our study occurred during delay conditioning, a procedure that is not affected by hippocampal lesions (Moyer et al., 1990; Weiss et al., 1999). Similarly, the stimulation in hippocampal ERKs reported by Atkins et al. (1998) occurred not only for hippocampal-dependent (cued) but also for hippocampal-independent (contextual) fear conditioning. The intraventricular injection of PD98059 produced only a small and marginally significant retardation of learning, although it blocked the learning-associated activation of ERK in both the hippocampus and vermis. Thus activation of ERKs does not appear to be importantly involved in acquisition of delay eyeblink conditioning. However, inhibition of ERKs has been shown to be associated with a blockade of learning and memory in other learning paradigms such as spatial learning and fear conditioning (Atkins et al., 1998; Blum et al., 1999; Schafe et al., 1999; Selcher et al., 1999).

\section{Significance of ERK and p38 MAPK activation in the cerebellar vermis}

The learning-dependent bilateral increase in both ERKs and p38 MAPK in the anterior vermis of the cerebellum represents a novel finding. The intraventricular injection of SB203580 produced a robust impairment of learning that was not related to an impairment in performance variables. We examined the possibility of a performance deficit by allowing drug washout after the third conditioning session and then examining CR production during an extinction session without any drug injections. If the animals had been learning but were incapable of producing CRs because of performance impairment, then one would expect that the percentage of CRs would increase during our extinction test because the learning could now be expressed in the absence of any performance deficit, but, as shown in Table 1, this was not the case. In previous experiments we had used this approach to distinguish between disruptive effects on performance versus retardation of associative learning (Welsh and Harvey, 1991, 1998). In addition, the lack of any significant effect of SB203580 on UR amplitudes suggests that sensory input and output of the brainstem NM eye-blink motor centers were not affected.

SB203580, a selective inhibitor of p38 MAPK, not only produced a significant retardation of learning but also blocked the learning-related activation of p38 MAPK in the anterior vermis.
This finding suggests that the activation of p38 MAPK in the vermis may be importantly involved in associative learning. Our finding of a critical role of the anterior vermis in eye-blink conditioning may be relevant to several recent studies that have suggested an important role for the cerebellar vermis in cognitive and affective processes. For example, human eye-blink conditioning was found to produce a learning-related activation of the cerebellum, with the correlation between metabolic activation and learning $(0.82 ; \mathrm{p}<0.001)$ being the highest for the anterior vermis (Logan and Grafton, 1995). Deficits in associative processes as seen in autism (Courchese, 1999; Levitt et al., 1999) and schizophrenia (Nopoulos et al., 1999) have been associated with abnormal vermal morphology. Finally, abnormalities of MAPKs have been reported in the cerebellar vermis of schizophrenic subjects (Kyosseva et al., 1999).

The absence of any changes in MAPKs in hemispheral lobule $\mathrm{VI}$ is consistent with the fact that its destruction has only minor effects on the acquisition of eye-blink conditioning in the rabbit (Lavond and Steinmetz, 1989; Harvey et al., 1993). Both chronic and reversible lesions of the inferior olivary nucleus have been reported to eliminate eye-blink conditioning in the rabbit (Yeo et al., 1986; Welsh and Harvey, 1998). However, the absence of any changes in MAPKs in the inferior olive may be caused by the fact that the brainstem sample containing the inferior olive was heterogeneous and thus the critical area (the dorsal accessory olivary nucleus) could have been too diluted to detect any changes.

Early studies had suggested a cerebellar involvement in autonomic conditioning. Thus removal of the cerebellum was shown to impair performance of salivary, cardiac, and respiratory conditioning (Orbeli, 1940; Livshits, 1947; Krasusky, 1957; Karamyan, 1959). More recent studies have demonstrated that these effects can be localized to the cerebellar vermis. For example, lesions of the anterior cerebellar vermis in the rabbit impair the acquisition of classically conditioned bradycardia in the rabbit (Supple and Kapp, 1993). It is possible, as suggested by Logan and Grafton (1995), that classical conditioning of the eye-blink response may be associated with significant autonomic conditioning. Although it is known that cardiac conditioning does not occur at short interstimulus intervals such as the $200 \mathrm{msec}$ used in this study (Powell and Levine-Bryce, 1988), we cannot exclude the possibility that other autonomic components including the aversive properties of the CS may have been conditioned (Swank, 2000a).

The major finding of this study is the demonstration of an involvement of p38 MAPK in associative learning. To our knowledge, this is the first report of such a role for p38 MAPK. It was thought originally that p38 MAPK, like JNK, was a stressactivated MAP kinase that played a key role in the regulation of cell apoptosis. However, a more extensive role for p38 MAPK has been suggested recently by the fact that growth factors, G-protein-coupled receptor agonists, and neurotransmitters are also able to regulate p38 MAPK activity (Zhen et al., 1998; Nebreda and Porras, 2000). Furthermore, accumulating evidence indicates that this kinase may play an important role in the regulation of cellular differentiation, proliferation, and function (Nebreda and Porras, 2000). It should be noted that, like the ERKs, p38 MAPK is enriched in the CNS (Ortiz et al., 1995; Zhen et al., 1999; Lee et al., 2000). In the cerebellum, Purkinje cells express high levels of p38 MAPK. Previous work demonstrated an age-related defect in p38 MAPK in rat brains, and caloric restriction was shown to modulate this change, suggesting that p38 MAPK may be involved in cellular mechanisms of aging 
of the brain (Zhen et al., 1999). If so, then p38 MAPK might be important in age-related cognitive deficits. The molecular mechanism by which p38 MAPK may promote neuronal plasticity underlying learning and memory is not clear. Transcriptional factors such as cAMP response element-binding protein (CREB) have been shown to play an essential role in cerebellar synaptic plasticity that is associated with eye-blink conditioning (Kim and Thompson, 1997), and MAPKs have been demonstrated to regulate a number of transcriptional factors such as Elk-1 and CREB (Bailey et al., 1997; Cammarota et al., 2000). Whether p38 MAPK or ERKs in the cerebellum regulate neuronal plasticity via CREB or other transcriptional factors remains to be elucidated. In addition, there is a need to identify the upstream signals that are responsible for the activation of MAPKs.

In conclusion, the activation of p38 MAPK in the anterior cerebellar vermis appears to play an important role in associative learning and may be the initiator of the transcriptional events that lead to memory consolidation.

\section{REFERENCES}

Aloyo VJ, Romano AG, Harvey JA (1993) Evidence for an involvement of the mu-type of opioid receptor in the modulation of learning. Neuroscience 55:511-519.

Atkins CM, Selcher JC, Petraitis JJ, Trzaskos JM, Sweatt JD (1998) The MAPK cascade is required for mammalian associative learning. Nat Neurosci 1:602-609.

Bailey CH, Kaang BK, Chen M, Martin KC, Lim CS, Casadio A, Kandel ER (1997) Mutation in the phosphorylation sites of MAP kinase blocks learning-related internalization of apCAM in Aplysia sensory neurons. Neuron 18:913-924.

Berman DE, Hazvi S, Rosenblum K, Seger R, Dudai Y (1998) Specific and differential activation of mitogen-activated protein kinase cascade by unfamiliar taste in the insular cortex of the behaving rat. J Neurosci 18:10037-10044.

Blum S, Moore AN, Adams F, Dash PK (1999) A mitogen-activated protein kinase cascade in the CA1/CA2 subfield of the dorsal hippocampus is essential for long-term spatial memory. J Neurosci 19:3535-3544.

Cammarota M, Bevilaqua LR, Ardenghi P, Paratcha G, Levi de Stein M, Izquierdo I, Medina JH (2000) Learning-associated activation of nuclear MAPK, CREB and Elk-1, along with Fos production, in the rat hippocampus after one-trial avoidance learning: abolition by NMDA receptor blockade. Brain Res Mol Brain Res 76:36-46.

Chen G, Steinmetz J (2000) Microinfusion of protein kinase inhibitor H7 into the cerebellum impaired the acquisition but not the retention of classical eyeblink conditioning in rabbits. Brain Res 856:193-201.

Courchese E (1999) An MRI study of autism: the cerebellum revisited. Neurology 52:1106-1107.

Davis RJ (1993) The mitogen-activated protein kinase signal transduction pathway. J Boil Chem 268:14533-14556.

Gormezano I, Kehoe EJ, Marshall BS (1983) Twenty years of classical conditioning with the rabbit. In: Progress in psychobiology and physiological psychology, Vol 10 (Epstein AN, ed), pp 197-275. New York: Academic.

Harvey JA (1987) Effects of drug on associative learning. In: Psychopharmacology, the third generation of progress (Meltzer HW, ed), pp 1485-1491. New York: Raven.

Harvey JA, Welsh JP, Yeo CH, Romano AG (1993) Recoverable and non-recoverable deficits in conditioned responses after cerebellar cortical lesions. J Neurosci 13:1624-1635.

Impey S, Obrietan K, Storm DR (1999) Making new connections: role of ERK/MAP kinase signaling in neuronal plasticity. Neuron 23:11-14.

Karamyan AI (1959) The evolution of corticocerebellar functional relationships. Zhurnal Vyshei Nervoi D'eyatel'nosti 5:346-444.

Kim JJ, Thompson RF (1997) Cerebellar circuits and synaptic mechanism involved in classical eyeblink conditioning. Trends Neurosci 20: 177-181.

Kornhauser JM, Greenberg ME (1997) A kinase to remember: dual roles for MAP kinase in long-term memory. Neuron 18:839-842.

Krasusky VK (1957) General nature of changes of food conditioned reflexes in dogs following a surgical lesion of the cerebellum. Zhurnal Vyshei Nervoi D'eyatel'nosti 3:733-740.

Kyosseva SV, Elbein AD, Griffin WS, Mrak RE, Lyon M, Karson CN
(1999) Mitogen-activated protein kinases in schizophrenia. Biol Psychiatry 46:689-696.

Lavond DG, Steinmetz JE (1989) Acquisition of classical conditioning without cerebellar cortex. Behav Brain Res 33:113-164.

Lee SH, Park J, Che Y, Han P-L, Lee J-K (2000) Constitutive activity and differential localization of $\mathrm{p} 38 \alpha$ and $\mathrm{p} 38 \beta$ MAPKs in adult mouse brain. J Neurosci Res 60:623-631.

Levitt JG, Blanton R, Capetillo-Cunliffe L, Guthrie D, Toga A, McCracken JT (1999) Cerebellar vermis lobules VIII-X in autism. Prog Neuropsychopharmacol Biol Psychiatry 23:625-633.

Livshits NN (1947) Effect of cerebellar extirpation on the conditioned reflexes of dogs. In: Trudy Fisiologicheskogo Instituta im Pavlova, Vol II (Champe GG, translator; Welsh JP, Harvey JA, eds), pp 11-50. Moscow: USSR Academy of Sciences.

Logan CG, Grafton ST (1995) Functional anatomy of human eyeblink conditioning determined with regional cerebral glucose-metabolism and positron-emission tomography. Proc Natl Acad Sci USA 92:7500-7504.

Morris RGM, Garrud P, Rawlins JNP, O'Keefe J (1982) Place navigation impaired in rats with hippocampal lesions. Nature 297:681-683.

Moyer Jr JR, Deyo RA, Disterhoft JF (1990) Hippocampectomy disrupts trace eye-blink conditioning in rabbits. Behav Neurosci 104:243-252.

Nebreda AR, Porras A (2000) p38 MAP kinases: beyond the stress response. Trends Biochem Sci 25:257-260.

Nopoulos PC, Ceilley JW, Gailis EA, Andreasen NC (1999) An MRI study of cerebellar vermis morphology in patients with schizophrenia: evidence in support of the cognitive dysmetria concept. Biol Psychiatry 46:703-711.

Orban PC, Chapman PF, Brambilla R (1999) Is the Ras-MAPK signalling pathway necessary for long-term memory formation? Trends Neurosci 22:38-44.

Orbeli LA (1940) New concepts concerning cerebellar function . Usp'ekhi Sovrem'ennoy Biologii 13:207-220.

Ortiz J, Harris HW, Guitart X, Terwilliger RZ, Haycock JW, Nestler EJ (1995) Extracellular signal-regulated protein kinase (ERKs) and ERK kinase (MEK) in brain: regional distribution and regulated by chronic morphine. J Neurosci 15:1285-1297.

Phillips RG, LeDoux JE (1994) Lesions of the dorsal hippocampal formation interfere with background but not foreground contextual fear conditioning. Learn Mem 1:34-44.

Powell DA, Levine-Bryce D (1988) A comparison of two model systems of associative learning: heart rate and eyeblink conditioning in the rabbit. Psychophysiology 25:627-682.

Romano AG, Bormann N, Harvey JA (1991) A unique enhancement of associative learning produced by methylenedioxyamphetamine. Behav Pharmacol 2:225-231.

Schafe GE, Nadel NV, Sullivan GM, Harris A, LeDoux JE (1999) Memory consolidation for contextual and auditory fear conditioning is dependent on protein synthesis, PKA, and MAP kinase. Learn Mem 6:97-110.

Selcher JC, Atkins CM, Trzaskos JM, Paylor R, Sweatt JD (1999) A necessity for MAP kinase activation in mammalian spatial learning. Learn Mem 6:478-490.

Supple Jr WF, Kapp BS (1993) The anterior cerebellar vermis: essential involvement in classically conditioned bradycardia in the rabbit. J Neurosci 13:3705-3711.

Swank MW (2000a) Pharmacological antagonism of tyrosine kinases and MAP kinase in brainstem blocks taste aversion learning in mice. Physiol Behav 69:499-503.

Swank MW (2000b) Phosphorylation of MAP kinase and CREB in mouse cortex and amygdala during taste aversion learning. NeuroReport 11:1625-1630.

Weiss C, Bouwmeester H, Power JM, Dsterhoft JF (1999) Hippocampal lesions prevent trace eyeblink conditioning in the freely moving rat. Behav Brain Res 99:123-132.

Welsh JP, Harvey JA (1991) Pavlovian conditioning in the rabbit during inactivation of the interpositus nucleus. J Physiol (Lond) 444:459-480.

Welsh JP, Harvey JA (1998) Acute inactivation of the inferior olive blocks associative learning. Eur J Neurosci 10:3321-3332.

Yeo CH, Hardiman MJ, Glickstein M (1986) Classical conditioning of the nictitating membrane response of the rabbit. IV. Lesions of the inferior olive. Exp Brain Res 63:81-92.

Zhen XC, Uryu K, Wang HY, Friedman E (1998) D1-dopamine receptor agonists mediate activation of p38 MAPK and JNK by a PKAdependent mechanism in SK-N-MC neuroblastoma cells. Mol Pharmacol 54:453-458.

Zhen XC, Cai GP, Uryu K, Johnson GP, Friedman E (1999) Ageassociated impairment in brain MAPK signal pathways and effect of caloric restriction in Fischer 244 rats. J Gerontol A Biol Sci Med Sci 54:B539-B548. 